\title{
Preliminary mechanisms of regulating PD-L1 expression in non-small cell lung cancer during the EMT process
}

\author{
FANG $\mathrm{LI}^{1,2^{*}}$, TENGJIAO ZHU ${ }^{3 *}$, YAO YUE $^{2}$, XIANG ZHU ${ }^{4}$, JIADONG WANG $^{2}$ and LI LIANG ${ }^{1}$ \\ ${ }^{1}$ Department of Tumor Chemotherapy and Radiation Sickness, Peking University Third Hospital, Beijing; \\ ${ }^{2}$ Department of Radiation Medicine, School of Basic Medical Science, Peking University, Beijing 100191; \\ ${ }^{3}$ Orthopedic Department, Peking University International Hospital, Beijing 102206; \\ ${ }^{4}$ Department of Pathology, Peking University Third Hospital, Beijing 100191, P.R. China
}

Received October 16, 2017; Accepted April 13, 2018

DOI: $10.3892 /$ or.2018.6474

\begin{abstract}
Non-small cell lung cancer (NSCLC) is one of the most severe malignant tumor types worldwide. Recent studies have reported an important role of PD-L1 in mediating immune evasion in the tumor microenvironment. In addition, increasing research has indicated that the expression of PD-L1 is related to the epithelial-mesenchymal transition (EMT) process, but the related mechanisms remain to be explored. In the present study, we explored the molecular mechanisms underlying the regulation of PD-L1 expression during the EMT process in NSCLC cells treated with transforming growth factor- $\beta 1$ (TGF- $\beta 1$ ) and fibroblast growth factor 2 (FGF2). The phenotypic alteration associated with EMT was evaluated by western blotting and confirmed by a wound-healing assay. The results revealed that EMT markedly promoted the expression of PD-L1 in the A549 cell line, while having no obvious influence on the H1650 and H1975 cells. Furthermore, the AKT pathway inhibitor LY294002, the ERK pathway inhibitor PD98059 and the TAK1 pathway inhibitor 5Z-7 inhibited the expression of PD-L1 in A549 and H1650 cells, but not in H1975 cells, during the EMT process. Moreover, our study indicated that the AKT, ERK and TAK1 pathways regulated the expression of PD-L1 by mediating transportation of the transcription factor Stat3 and the p65 subunit of $N F-\kappa B$ from the cytoplasm to the nucleus, with such findings determined by western blotting
\end{abstract}

Correspondence to: $\mathrm{Dr} \mathrm{Li}$ Liang, Department of Tumor Chemotherapy and Radiation Sickness, Peking University Third Hospital, 49 North Garden Road, Haidian, Beijing 100191, P.R. China E-mail: liang.dr@163.com

Dr Jiadong Wang, Department of Radiation Medicine, School of Basic Medical Science, Peking University, 30 Xueyuan Road, Haidian, Beijing 100191, P.R. China

E-mail: wangjd@bjmu.edu.cn

*Contributed equally

Key words: NSCLC, PD-L1, EMT and flow cytometric analyses. Furthermore, the expression of PD-L1 was significantly increased following treatment with gefitinib in a tumor xenograft model. In summary, our results support the role of ERK, AKT and TAK1 in mediating the expression of PD-L1 during the EMT process, and indicate a promising strategy of PD-L1-targeted therapy for the clinical treatment of NSCLC.

\section{Introduction}

Lung cancer is one of the most common and fatal malignant tumor types worldwide (1). Non-small cell lung cancer (NSCLC) accounts for 80-85\% of all lung cancer cases (2). Since 2003, three generations of TKI drugs have received FDA approval for the clinical treatment of NSCLC (3). However, the increasing rate of drug resistance in patients treated with molecular targeted therapy is becoming a severe challenge for treatment. Moreover, even patients who exhibit sensitivity to targeted drugs at the start of treatment can become resistant within a few months $(4,5)$. The molecular mechanisms of drug resistance, regarding both mutational and non-mutational pathways, have only recently begun to be fully explored (6); however, at present, accumulating evidence indicates that epithelial-mesenchymal transition (EMT) plays an important role in the resistance to targeted therapy in a non-mutational manner (7).

EMT is a process by which epithelial cells are converted into mesenchymal cells. This involves a phenotypic switch of cellular characteristics, whereby cells lose their adhesive properties and cell-cell contacts while acquiring migratory properties, which can promote tumor progression. The process of EMT is mediated by a change in the expression of cell surface proteins and the induction of cytoskeletal rearrangement, among other phenotypic alterations $(8,9)$. Diverse signaling molecules can trigger EMT, including hepatocyte growth factor (HGF), epidermal growth factor (EGF), fibroblast growth factor (FGF) $(10,11)$. In particular, transforming growth factor- $\beta 1$ (TGF- $\beta 1$ ) is considered to be a key regulator in the EMT pathway (12). TGF- $\beta 1$ belongs to a family of related polypeptide factors with shared structural motifs, which serve vital roles in processes such as cell growth, differentiation and oxidative stress (13). TGF- $\beta$-mediated EMT has 
been identified in many different tumor types, including lung cancer (14). TGF- $\beta$ promotes EMT-related gene transcription in a Smad3-dependent manner through the $\beta$-integrin signal transduction pathway or the mitogen-activated protein kinase (MAPK) signaling pathway, in order to influence gene expression and ultimately transform epithelial cells from an epithelial phenotype to a stromal cell phenotype, thus promoting the occurrence of EMT (15-17).

Programmed death-1 (PD-1) is so named due to its association with apoptosis, and is mainly expressed in CD4+ and CD8+ T lymphocytes, B lymphocytes, NK (natural killer) cells and regulatory T cells (Tregs) $(18,19)$. The two ligands of PD-1 are PD-L1 (also known as CD274 or B7-H1) and PD-L2 (also known as CD273 or B7-DC) (19). Cytokines, such as IL-4 and IL-10, activate the PD-1/PD-L1 pathway, which affects T cells with high expression of PD-1 molecules on their surface or tumor cells with high surface-expression of PD-L1 $(20,21)$. As a result, $T$ cell function is inhibited and the immune system is suppressed in its recognition and attack of tumor cells. Recent studies have indicated that the EMT in tumor cells is associated with immune escape, which results in insensitivity to targeted drugs $(22,23)$

In this study, we first speculated on the effect of the EMT process induced by TGF- $\beta 1$ and FGF2 on the expression of PD-L1 in the NSCLC cell lines A549 (wild-type), H1650 (E746_A750 deletion) and H1975 (harboring the mutation of T790 in EGFR). We then investigated the molecular mechanisms underlying the regulation of PD-L1 expression during the EMT process, by treating the three NSCLC cell lines with the AKT pathway inhibitor Ly294002, the ERK pathway inhibitor PD98059 and the TAK1 pathway inhibitor 5Z-7. Furthermore, we assessed the expression of PD-L1 in the NSCLC cells following treatment with EGFR-TKI (gefitinib). Collectively, these tests aimed to provide a theoretical basis for the development of new methods to reverse drug resistance in NSCLC.

\section{Materials and methods}

Reagents. Antibodies against E-cadherin (cat. no. 14472), $\mathrm{N}$-cadherin (cat. no. 14215), vimentin (cat. no. 5741), mTOR (cat. no. 2983), phospho-mTOR (Ser 2448) (cat. no. 5536), Akt (cat. no. 4685), phospho-Akt (Ser473) (cat. no. 4060), p44/42 MAPK (Erk1/2) (cat. no. 4695), Phospho-p44/42 MAPK (Erk1/2) (Thr202/Tyr204) (cat. no. 4370), TAK1 (cat. no. 4505), p65 (cat. no. 8242), GADPH (cat. no. 5174) and human Basic Fibroblast Growth Factor (hFGF basic/FGF2) (cat. no. 8910) were obtained from Cell Signaling Technology, Inc. (Danvers, MA, USA). PD-L1 (cat. no. 205921) was purchased from Abcam (Cambridge, MA, USA). Gefitinib (cat. no. S1025), LY294002 (cat. no. S1105) and PD98059 (cat. no. S1177) were purchased from Selleck Chemicals (Houston, TX, USA) and stock solution was prepared in dimethyl sulfoxide (DMSO) at $10 \mathrm{mM}$. 5Z-7-oxozeaenol (5Z-7) was obtained from Sigma-Aldrich (Rehovot, Israel) and dissolved in DMSO. Recombinant human TGF- $\beta 1$ was purchased from PeproTech (cat.no. 100-21C; Rocky Hill, NJ, USA). Rhodamine phalloidin and DAPI were obtained from Sigma-Aldrich. PE-conjugated anti-Human PD-L1 (cat. no. 12-5983-41) was purchased from eBioscience/Thermo Fisher Scientific (San Diego,
CA, USA). NE-PER ${ }^{\mathrm{TM}}$ Nuclear and Cytoplasmic Extraction Reagents were obtained from Thermo Fisher Scientific ${ }^{\mathrm{TM}}$ (Rockford, IL, USA).

Cell lines. Non-small cell lung cancer cell lines A549, NCI-H1975 and NCI-H1650 were purchased from the China Infrastructure of Cell Line Resources (Beijing, China). The cells were grown in McCoy's medium (Gibco; Invitrogen, Carlsbad, Calif) or RPMI-1640 modified medium (HyClone; GE Healthcare Life Sciences, Logan, UT, USA) supplemented with $10 \%$ fetal bovine serum (Gibco; Invitrogen, Carlsbad, Calif), $100 \mathrm{U} / \mathrm{ml}$ penicillin and $100 \mu \mathrm{g} / \mathrm{ml}$ streptomycin. The cells were maintained in humidified air containing $5 \% \mathrm{CO}_{2}$ at $37^{\circ} \mathrm{C}$ in a monolayer culture.

Wound healing assay. Cells $\left(5 \times 10^{5}\right)$ in 6 -well plates were allowed to grow until reaching $80 \%$ confluency. After starvation overnight, wounds were scratched on the cell surface using a $100 \mu \mathrm{l}$ micropipette tip. The cells were treated with $10 \mathrm{ng} / \mathrm{ml} \mathrm{TGF}-\beta 1,10 \mathrm{ng} / \mathrm{ml}$ FGF2 or $10 \mathrm{ng} / \mathrm{ml}$ TGF- $\beta 1$ plus $10 \mathrm{ng} / \mathrm{ml} \mathrm{FGF} 2$ for $48 \mathrm{~h}$. The changes of the wound area were captured by Nikon camera.

Immunofluorescence staining. Cells $5 \times 10^{5}$ were seeded on 6-well plates coated with coverslips in McCoy's medium or RPMI-1640 medium. Following starvation overnight in serum-free medium, the cells were treated with $10 \mathrm{ng} / \mathrm{ml}$ TGF- $\beta 1$ plus $10 \mathrm{ng} / \mathrm{ml}$ FGF2 for $48 \mathrm{~h}$. The cells were fixed for $20 \mathrm{~min}$ in paraformaldehyde and permeabilized in $0.2 \%$ Triton X-100 for $5 \mathrm{~min}$. Then, F-actin was stained with Rhodamine phalloidin and the nuclei with DAPI. The images were captured using a Leica SP5 spectral confocal microscope.

Western blot analysis. The cells in 6-well plates were washed with cold phosphate-buffered saline (PBS) twice and lysed with $80 \mu$ l RIPA solution containing $1 \mathrm{mM}$ PMSF in each well. Lysates were incubated on ice and vortexed every $5 \mathrm{~min}$, in total $30 \mathrm{~min}$. Then the supernatant was collected after centrifugation $\left(15,000 \mathrm{x} \mathrm{g}\right.$ for $5 \mathrm{~min}$ at $\left.4^{\circ} \mathrm{C}\right)$. The concentration of the total protein was assessed using the BCA Protein Assay Kit (Beijing Solarbio Science \& Technology Co., Ltd., Beijing, China). Total protein (35 $\mu \mathrm{g}$ ) was subjected to $10-12 \%$ SDS-PAGE and blotted on PVDF membranes (EMD Millipore, Bedford, MA, USA). Then the membranes were incubated with primary antibodies mentioned above (1:1,000 dilution) overnight at $4^{\circ} \mathrm{C}$. The results were detected using the Odyssey detection system (LI-COR Biosciences, Lincoln, NE, USA).

Flow cytometric analysis. Cells were plated in 6-well plates $\left(1-5 \times 10^{5}\right)$ and treated with $0.05 \%$ trypsin. Then, the cells were harvested by centrifugation ( $800 \mathrm{x} \mathrm{g}, 5 \mathrm{~min})$. Flow cytometric cells were stained with the PE-conjugated anti-human PD-L1 clone M1H1. The cells were assessed using Accuri ${ }^{\mathrm{TM}}$ C6 (BD Biosciences, Franklin Lakes, NJ, USA). The data was analyzed using FlowJo software (TreeStar, Inc., Ashland, OR, USA) and the PD-L1 levels were determined by calculating the Median Fluorescence Intensity (MFI).

Nuclear translocation analysis. Cells were harvested with trypsin-EDTA, and then centrifuged at $500 \mathrm{x} \mathrm{g}$ for $5 \mathrm{~min}$. The 
A

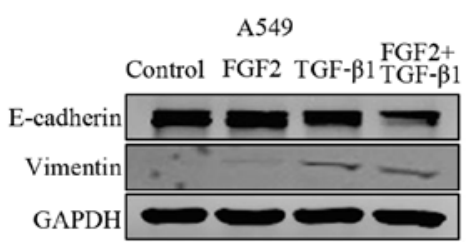

D

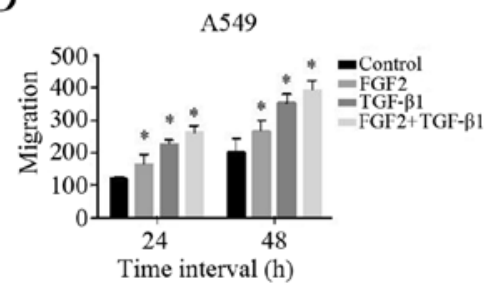

G

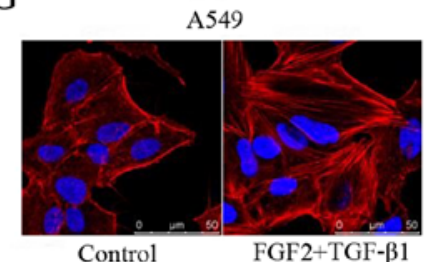

B

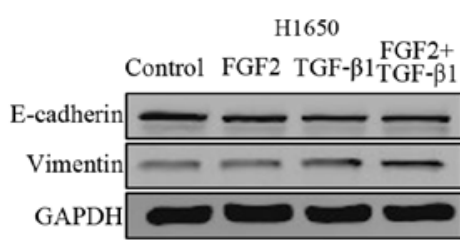

E

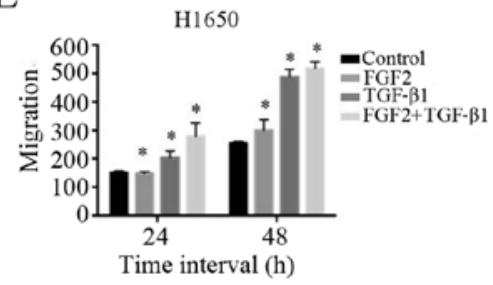

H

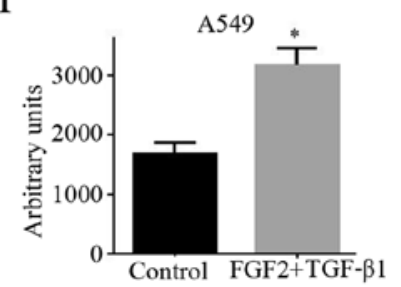

C

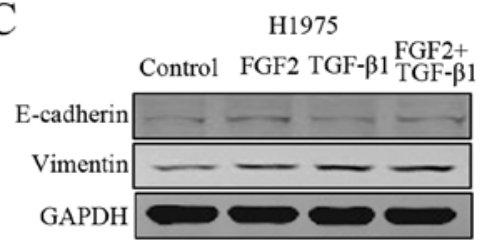

F

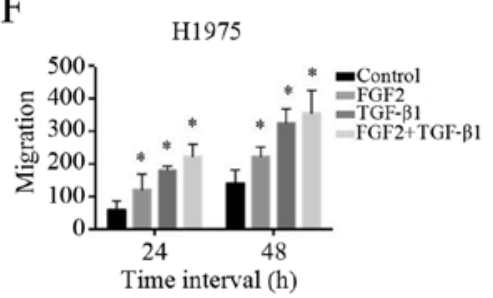

Figure 1. The formation of the EMT phenotype induced by FGF2 plus TGF- $\beta 1$ in three NSCLC cell lines. (A-C) Cells were starved in serum-free medium overnight and cultured with FGF2, TGF- $\beta 1$ or FGF2 plus TGF- $\beta 1$ for 48 h. The expression of E-cadherin and vimentin/N-cadherin were analyzed by western blotting. The blots represent one of three independent experiments. (D-F) Cells were starved in serum-free medium overnight, after which a scratch was created in the cell monolayer. FGF2, TGF- $\beta 1$ or FGF2 plus TGF- $\beta 1$ were then added. The migration of the cells was evaluated at 24 and 48 h. (G) After starvation overnight, A549 cells were incubated with FGF2 plus TGF- $\beta 1$ for 48 h, after which the cells were stained with rhodamine-phalloidin (red actin fibers) and DAPI (blue) and images were captured with a confocal microscope. Scale bar, $50 \mu \mathrm{M}$. (H) The histogram revealed the quantification of cell size. * $<0.05$ vs. the control group.

cells were then transferred to a $1.5 \mathrm{ml}$ microcentrifuge tube and pelleted by centrifugation at $500 \mathrm{x} \mathrm{g}$ for $2-3 \mathrm{~min}$. A pipette was used to carefully remove and discard the supernatant, leaving the cell pellet as dry as possible. Ice-cold CER I was added to the cell pellet. The tube was then vortexed vigorously on the highest setting for $15 \mathrm{sec}$ to fully suspend the cell pellet and subsequently the tube was incubated on ice for $10 \mathrm{~min}$. Ice-cold CER II was added to the tube and then the tube was vortexed for $5 \mathrm{sec}$ on the highest setting. Incubation followed on ice for $1 \mathrm{~min}$ and then the tube was centrifuged for $5 \mathrm{~min}$ at a maximum speed $(\sim 16,000 \mathrm{x} \mathrm{g})$. The supernatant was then immediately transferred to a clean pre-chilled tube and vortexed on the highest setting for $15 \mathrm{sec}$ in ice-cold NER. Subsequently, the sample was placed on ice and vortexing continued for $15 \mathrm{sec}$ every $10 \mathrm{~min}$, for a total of $40 \mathrm{~min}$. The tube was then centrifuged at maximum speed $(\sim 16,000 \mathrm{xg})$ in a microcentrifuge for $10 \mathrm{~min}$. Finally, the supernatant (nuclear extract) fraction was immediately transferred to a clean pre-chilled tube and the extracts were stored at $-80^{\circ} \mathrm{C}$ until use.

Animals and tumor xenograft experiments. As mentioned in our previous study (24), total 12 five- to six-week-old female $\mathrm{BALB} / \mathrm{c}$ nude mice were raised under specific pathogen-free

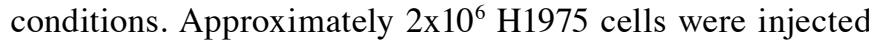
subcutaneously into the right flank region of nude mice. The tumor volume was assessed every three days. Mice bearing tumors $\sim 50 \mathrm{~mm}^{3}$ in volume were randomized into six mice per group $(n=6)$. The two group were administrated every day for
21 days by oral gavage as followed: (a) saline; (b) gefitinib. At harvest, the mice were sacrificed and the tumor tissues were fixed in formalin for immunohistochemistry.

Immunohistochemistry. A series of 3- $\mu$ m sections were obtained from each paraffin block. After heat immobilization, deparaffinization and rehydration, the sections were treated with $10 \mathrm{mM}$ sodium citrate buffer at $\mathrm{pH} 6.0$ in boiling temperature for antigen retrieval. Then the tissue sections were incubated with the primary antibodies PD-L1 (1:100) overnight at $4{ }^{\circ} \mathrm{C}$ and incubated with the secondary antibody biotin (cat. no. 926-32211; (LI-COR Biosciences) for $20 \mathrm{~min}$ at room temperature. Targeted proteins PD-L1 were visualized using peroxidase substrate diaminobenzidine (DAB). Staining intensities were estimated in five random fields per section by three independent observers individually using microscope equipped with camera.

Statistics. Data were computed using Student's t-test (two-tailed) or one-way ANOVA analysis. All values were presented as the mean $\pm \mathrm{SD}$. $\mathrm{P}<0.05$ was considered to indicate a statistically significant difference.

\section{Results}

FGF2 plus TGF- $\beta 1$ induced EMT in NSCLC cell lines. In this study, we explored the effects of FGF2 plus TGF- $\beta 1$ on promoting the EMT process in three NSCLC cell lines: A549 
A

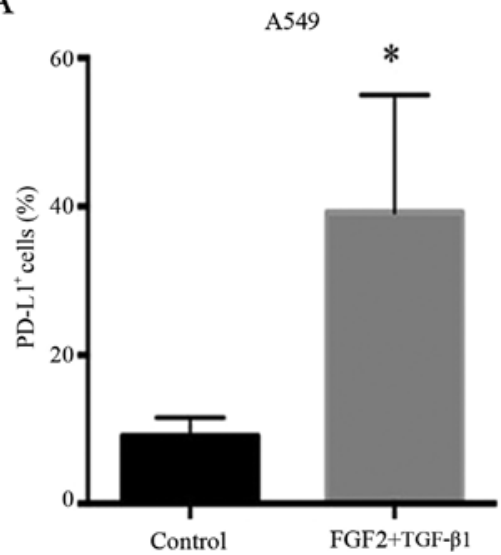

B

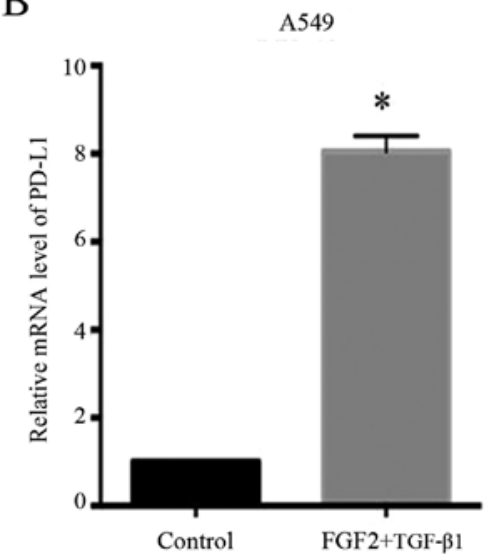

Figure 2. EMT promotes the expression of PD-L1 in the A549 cell line. (A) After being cultured with FGF2 plus TGF- $\beta 1$ for 48 h, the expression of PD-L1 was detected by flow cytometry (the histogram plots is listed in the last page). (B) The expression of PD-L1 after the same treatment was analyzed by RT-PCR ${ }^{*} \mathrm{P}<0.05$ vs. the control group from three independent experiments.

A

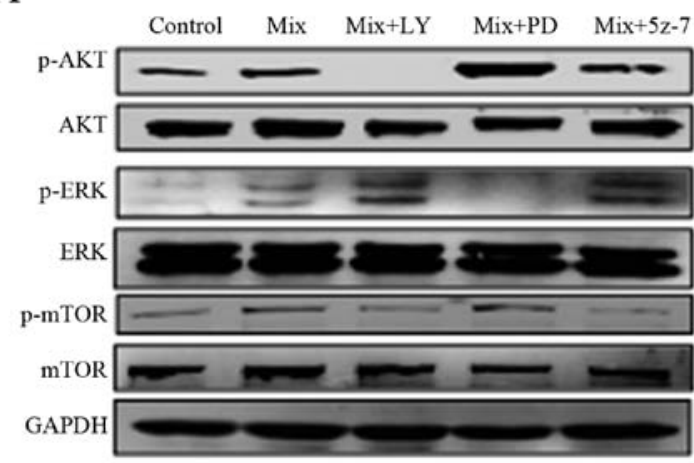

B

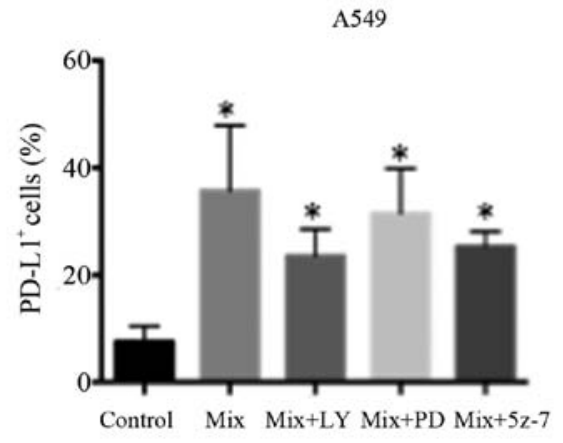

$\mathrm{C}$

Cytoplasm

Nucleus

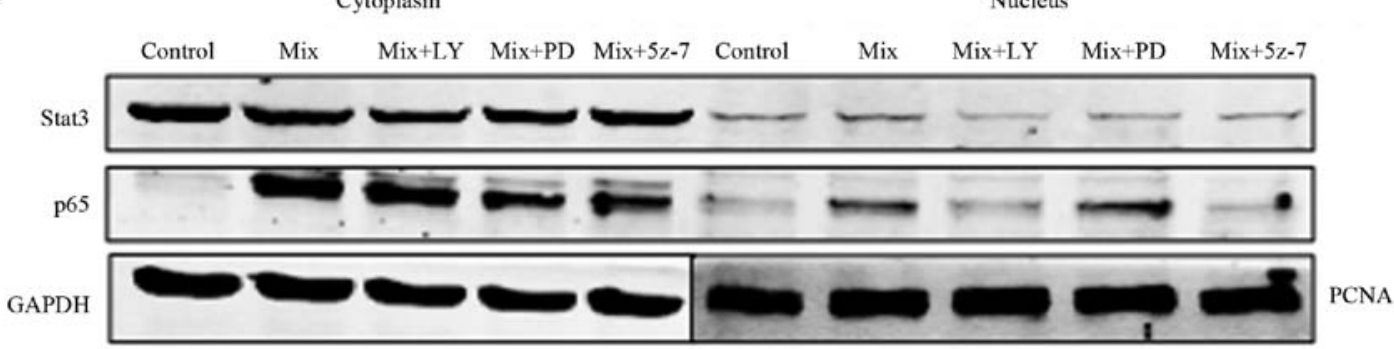

Figure 3. AKT, ERK and TAK1 pathways play an important role in mediating PD-L1 expression during the EMT process. (A) A549 cells were treated with the AKT inhibitor LY294002, the ERK inhibitor PD98059 and the TAK1 inhibitor 5Z-7 after induction of the EMT process for $48 \mathrm{~h}$ and the related proteins were detected by western blotting. (B) The expression of PD-L1 after treatment was analyzed by flow cytometry. (C) Cytoplasm and nuclear protein extracts were subjected to western blot analysis; the expression levels of $\mathrm{p} 65$ and Stat 3 in the nucleus and cytoplasm were detected separately. All data are representative of three independent experiments. $\mathrm{P}<0.05$ vs. the control group.

(wild-type EGFR), H1650 (E746_A750 deletion) and H1975 (T790M). We stimulated the cells with TGF- $\beta 1$, FGF2 or FGF2 plus TGF- $\beta 1$ for $48 \mathrm{~h}$ in serum-free medium. The EMT phenotype was confirmed in the FGF2 plus TGF- $\beta 1$ group when the expression of E-cadherin (an epithelial marker) was decreased and the expression of vimentin and $\mathrm{N}$-cadherin (mesenchymal markers) were markedly increased compared with the other groups (Fig. 1A-C). Subsequently, cell migration capacity was assessed by a wound healing assay. We observed that the migration ability of cells treated with FGF2 plus TGF- $\beta 1$ was enhanced in comparison with the other groups at both 24 and
$48 \mathrm{~h}$ (Fig. 1D-F). These results indicated that treatment with FGF2 plus TGF- $\beta 1$ downregulated E-cadherin, upregulated vimentin and $\mathrm{N}$-cadherin and increased migration ability in all three NSCLC cell lines.

In addition to affecting cell migratory ability and EMT-related protein expression, morphological changes further confirmed the process of EMT. An increase in cell size mediated by FGF2 plus TGF- $\beta 1$ was one of the predominant characterizations of EMT. As shown in Fig. $1 \mathrm{G}$ and $\mathrm{H}$, the A549 cells treated with FGF2 plus TGF- $\beta 1$ for 48 h were markedly enlarged compared with the control cells. Collectively, 
A

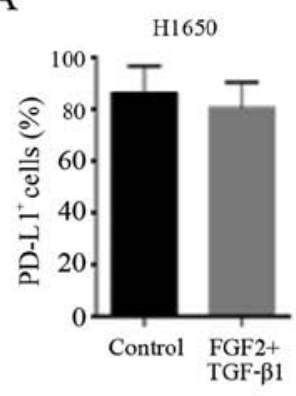

D

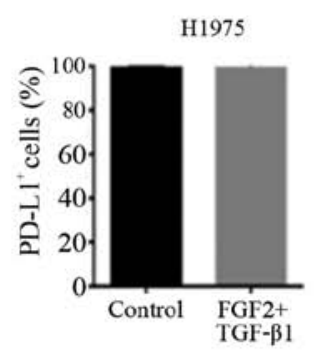

B

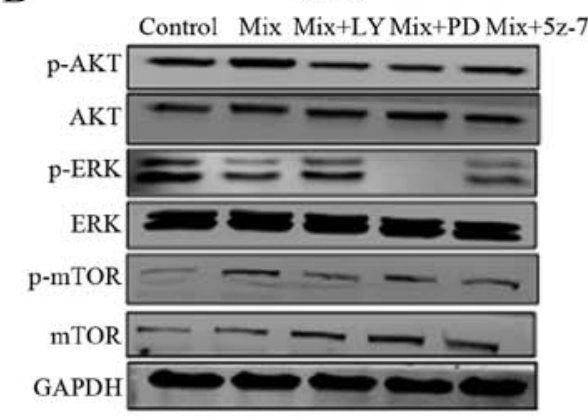

E

$\mathrm{H} 1975$

Control Mix Mix+LYMix+PD Mix $+5 z-7$

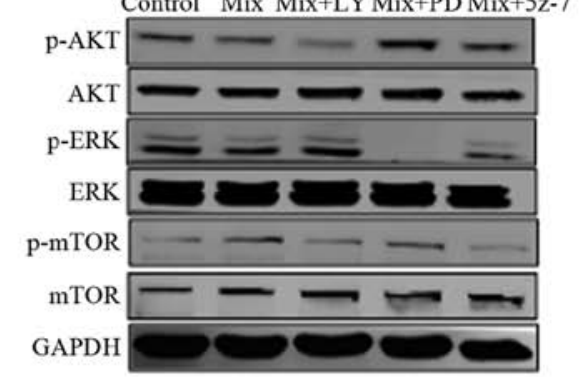

C

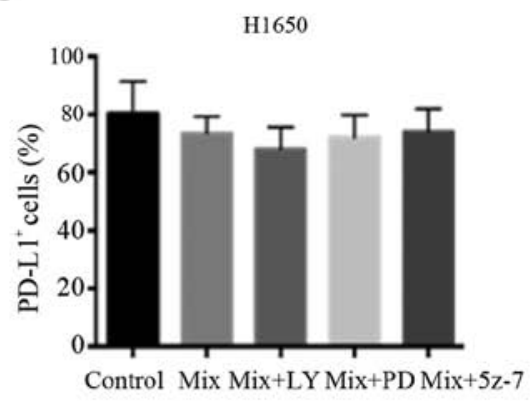

F

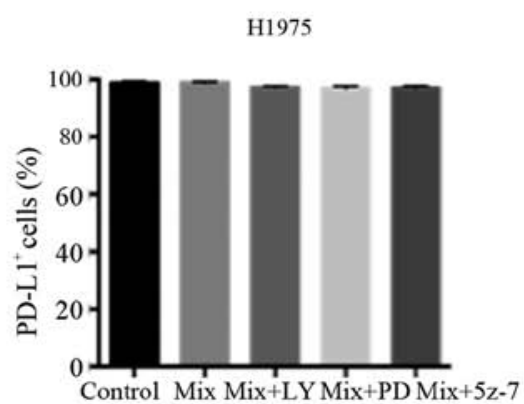

Figure 4. Related signaling pathways regulate PD-L1 expression in H1650 but not H1975 cells during the EMT process. (A) Analysis of PD-L1 expression after FGF2 plus TGF- $\beta 1$ treatment in H1650 cells. (B) H1650 cells were treated with the AKT inhibitor LY294002, the ERK inhibitor PD98059 and the TAK1 inhibitor 5Z-7 after induction of the EMT process for $48 \mathrm{~h}$ and the related proteins were detected by western blotting. (C) The expression of PD-L1 after treatment was analyzed by flow cytometry. (D) Analysis of PD-L1 expression after FGF2 plus TGF- $\beta 1$ treatment in H1975 cells. (E) H1975 cells were treated with the AKT inhibitor LY294002, the ERK inhibitor PD98059 and the TAK1 inhibitor 5Z-7 after induction of the EMT process for $48 \mathrm{~h}$ and the related proteins were detected by western blotting. (F) The expression of PD-L1 after treatment was analyzed by flow cytometry. All data are representative of three independent experiments. ${ }^{*}<0.05$ vs. the control group.

these results indicated that FGF2 plus TGF- $\beta 1$ treatment is an effective way of promoting the induction of a physiological EMT phenotype.

EMT promotes the expression of $P D-L 1$ in the wild-type EGFR NSCLC cell line. The expression of PD-L1 in A549 cells was markedly increased during the EMT process induced by FGF2 plus TGF- $\beta 1$ treatment. As shown in Fig. $2 \mathrm{~A}$, the PD-L1-positive staining on the surface of A549 cells was significantly increased after treatment with FGF 2 plus TGF- $\beta 1$ as determined by flow cytometric analysis $(\mathrm{P}=0.0029)$. Moreover, this result was further confirmed by RT-PCR analysis. The mRNA level of PD-L1 during the EMT process was higher than that in the control cells $(\mathrm{P}=0.009)$ (Fig. 2B). These results indicated that the EMT process markedly promoted the expression of PD-L1 in A549 cells.

Related signaling pathways during the EMT process in wild-type EGFR A549 cells. To further explore the molecular mechanisms of EMT-induced PD-L1 expression, we assessed several EMT-related signaling pathways. As shown in Fig. 3A, the cells were treated with the AKT inhibitor LY294002, the ERK inhibitor PD98059 and the TAK1 inhibitor 5Z-7 after the induction of EMT. Following treatment, we observed that the expression of phosphorylated AKT, phosphorylated mTOR, phosphorylated ERK was inhibited compared to FGF2 plus TGF- $\beta 1$ treatment. Furthermore, the expression of PD-L1 was decreased following treatment with the inhibitors (Fig. 3B). According to previous research, phosphorylated proteins mediate downstream transcription factors in their signaling pathways. Our study revealed that the inhibitors of AKT, ERK and TAK1 reduced the expression of Stat 3 in the nucleus to a similar extent. Additionally, treatment with AKT inhibitor (LY294002) and TAK1 inhibitor (5Z-7), but not ERK inhibitor (PD98059), elicited decreased nuclear expression of p65 (Fig. 3C). The results confirmed our hypothesis that inhibition of AKT, ERK and TAK1 phosphorylation could inhibit the import of Stat 3 transcription factor into the nucleus, and thus inhibit the expression. Furthermore, our results confirmed that inhibition of AKT and TAK1 phosphorylation could further inhibit the nuclear import of the p65 subunit of NF- $\kappa B$, thus indicating its synergistic role in influencing the expression of PD-L1.

Related signaling pathways regulate PD-L1 expression in H1650 cells while having no influence on H1975 cells during the EMT process. In this study, we further explored the regulatory mechanism underlying PD-L1 expression in the H1650 cell line. As shown in Fig. 4A, the expression of PD-L1 during the EMT process underwent no significant change in the H1650 cells. And the expression of relative proteins was reduced after the treatment of inhibitors (Fig. 4B). In addition, the inhibitors of AKT (LY294002), ERK (PD98059) and TAK1 (5Z-7) decreased the expression of PD-L1 (Fig. 4C).

In addition, we explored the influence of EMT on the T790M EGFR mutant cell line H1975. As shown in Fig. 4D, the EMT process had no influence on the expression of PD-L1 ( $\mathrm{P}=0.3739)$. Even following the inhibition of 
A
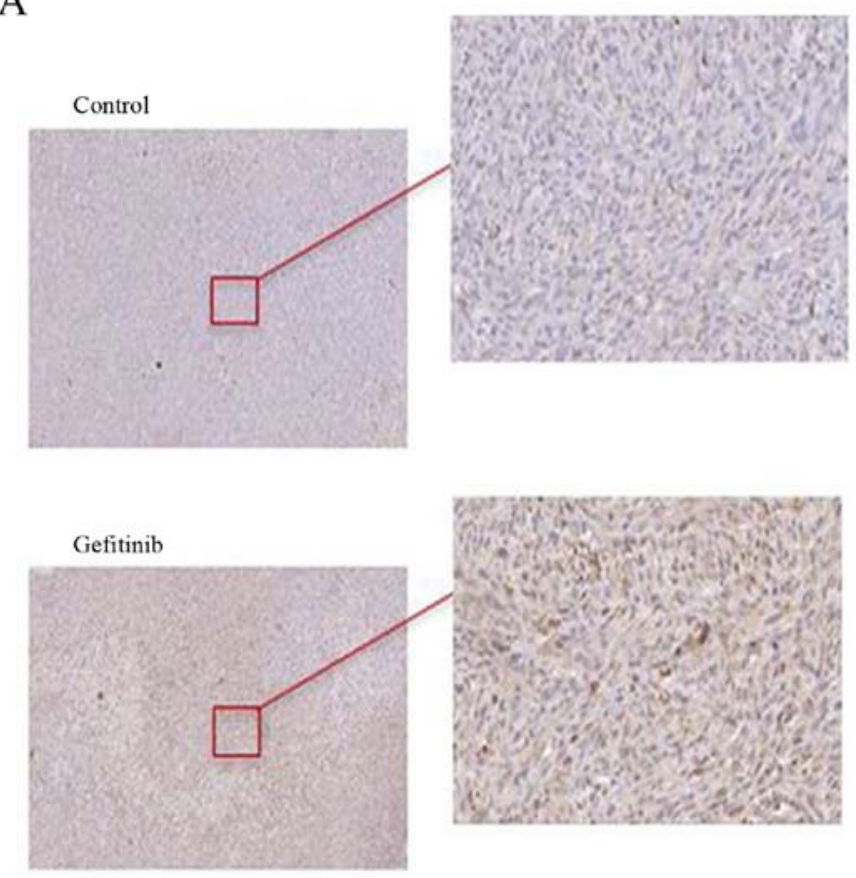

B

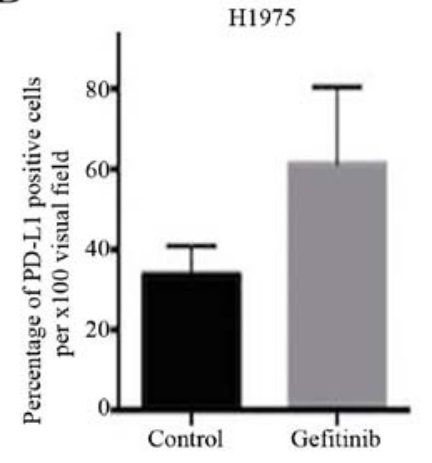

Figure 5. Gefitinib promotes the expression of PD-L1 in H1975 xenograft nude mice. (A and B) The expression of PD-L1 (Left panel x5, Right panel x20) in H1975 xenograft nude mice after gefitinib treatment was analyzed by IHC. $\mathrm{N}=6$.

relative proteins (Fig. 4E), the expression of PD-L1 had no changes (Fig. 4F). These results revealed that the signaling pathways of AKT, ERK and TAK1 served an important role in promoting the expression of PD-L1 during the EMT process in H1650 cells, but not in H1975 cells.

Gefitinib promotes the expression of PD-L1 in H1975 xenograft nude mice. In the present study, we also assessed the expression of PD-L1 in the tumor tissue of an H1975 xenograft nude mouse model. As shown in Fig. 5, the expression of PD-L1 in the gefitinib treatment group was markedly higher compared with that in the control group. This result revealed that gefitinib promoted the expression of PD-L1 in the H1975 xenograft nude mice.

\section{Discussion}

Currently, researchers are becoming increasingly aware that the occurrence and development of tumors are processes involving mutual antagonism between the tumor and hosT cells. The interaction between the tumor and host serves an important role in tumor progression and metastasis. Furthermore, the host immune system and the immune microenvironment are considered to be crucial determinants of tumor progression and metastasis. Under normal conditions, the immune system of the host body should be able to recognize and kill the malignanT cells to remove or control the growth of tumor tissue. However, the immune system of cancer patients is typically inhibited. One of the most obvious characteristics of this inhibition is the clustering of immune suppressor cells around the tumor mass in its microenvironment. PD-1/PD-L1 is one of the main pathways involved in tumor immune evasion. PD-L1 mRNA is widely expressed in normal human tissues and organs, while the PD-L1 protein is rarely expressed in normal human tissues, being limited to embryonic tonsil tissue, macrophage-like cells, lung cells and liver cells at a low level of expression (25). This phenomenon reveals that the expression of PD-L1 receives post-transcriptional regulation under normal physiological conditions. However, PD-L1 is abundantly expressed on the cell membranes of various tumor cells. Moreover, PD-L1 is expressed in tumor lesions, but not in metastatic cells in most tumors (25). These findings indicate that PD-L1 is selectively expressed in tumor tissues, and that its expression is closely related to the tumor microenvironment.

Lung cancer is one of the most common and fatal malignant tumor types worldwide. Non-small cell lung cancer (NSCLC) accounts for $80-85 \%$ of all lung cancer cases (26). Since 2003, three generations of TKI drugs have received FDA approval for the clinical treatment of NSCLC (27). However, the increasing rate of drug resistance in patients treated with chemotherapy increasingly becomes a serious challenge; even patients who are sensitive to chemotherapeutic drugs at the start of treatment develop resistance within a few months (28). The molecular mechanisms of drug resistance, including both mutational and non-mutational, have only recently begun to be explored (29-32). At present, accumulating evidence has indicated that the epithelial-mesenchymal transition (EMT) plays an important role in chemo-resistance in a non-mutational way. In the present study, we selected three NSCLC cell lines, A549 (wild-type EGFR), NCI-H1975 (L858R and T790M mutation; EGFR-TKI-resistant) and NCI-H1650 (E746_A750 deletion mutation; EGFR-TKI-sensitive), and explored the relationship between the expression of PD-L1 and the EMT process.

Numerous primary studies have demonstrated that EMT is strongly associated with cancer metastasis (33-34). Cadherin 
switching is a main characteristic alteration in several types of cancer, including NSCLC. The inactivation of the epithelial marker E-cadherin is the pivotal event of EMT in tumor progression (9). Furthermore, activation of certain mesenchymal markers, such as vimentin and $\mathrm{N}$-cadherin, may play a positive role in the process of metastasis since the normal cell-cell and cell-matrix contacts are disrupted (35). The aforementioned changes promote cell migration and contribute to loss of cell adhesion. A recent study suggested a relationship between PD-L1 expression and the EMT process in promoting the occurrence of immune evasion and metastasis progression (22). In this study, we created an effective way of promoting the induction of a physiological-like EMT phenotype in A549 cells. Additionally, our data elucidated the key role of EMT in triggering the expression of PD-L1 in the A549 cell line. We further explored the mechanisms of EMT in promoting the expression of PD-L1. The results indicated that the AKT, ERK and TAK1 pathways play synergistic roles in regulating the expression of PD-L1 during the EMT process, by mediating nuclear import of the transcription factor Stat 3 and the p65 subunit of NF- $\mathrm{NB}$ in A549 cells. These regulatory mechanisms may be applied to the EGFR-TKI sensitive cell line H1650, but not the EGFR-TKI resistant cell line H1975. Furthermore, gefitinib, an oral EGFR-TKI, increased the expression of PD-L1 in an H1975 xenograft tumor. Collectively, the aforementioned results revealed that EMT could mediate PD-L1 expression via multiple pathways, and thereby may serve an important role in immune evasion in the tumor microenvironment. However, our research is in the primary stage and further study is warranted to confirm the present conclusions.

In the clinical treatment of NSCLC, certain patients exhibit primary drug resistance to EGFR-TKI without having gene mutations. Considering the results of previous studies together with our findings leads us to make the assumption that EMT plays an important role in promoting the expression of PD-L1 in NSCLC cases expressing either wild-type EGFR or activated EGFR mutant by mediating the AKT-mTOR, ERK and TAK1-p65 pathways, but not in cases with EGFR-TKI resistance. Therefore, EMT may play a crucial role in primary drug resistance to EGFR-TKI in patients without mutation by promoting immune evasion, while having little effect on NSCLC with EGFR-TKI-resistant gene mutation (T790M). Although the process of tumor metastasis is regulated by various key factors, such as the circulatory system (26), the phosphorylation of Smad2 and Smad3 (37), and diverse growth factors in the tumor microenvironment (38), EMT may be one of the key cross-linking components mediating all of the necessary elements during the process of metastasis. Therefore, inhibition of EMT induction could be applied as a novel therapeutic method for the treatment of NSCLC harboring wild-type EGFR or EGFR-TKI-sensitizing mutation.

\section{Acknowledgements}

Not applicable.

\section{Funding}

This present study was supported by a grant from Peking University Third Hospital of China (no. BYSY2014011).

\section{Availability of data and materials}

The datasets used during the present study are available from the corresponding author upon reasonable request.

\section{Authors' contributions}

LF and ZTJ conceived and designed the study. LF, YY and XZ performed the experiments. LL and WJD reviewed and edited the manuscript. All authors read and approved the manuscript and agree to be accountable for all aspects of the research in ensuring that the accuracy or integrity of any part of the work are appropriately investigated and resolved.

\section{Ethics approval and consent to participate}

All experimental protocols were approved by the Institutional Review Board of the Department of Laboratory Animal Science of Peking university Health Science Center (Beijing, China).

\section{Patient consent for publication}

Not applicable.

\section{Competing interests}

The authors state that they have no competing interests.

\section{References}

1. Siegel RL, Miller KD and Jemal A: Cancer statistics, 2017. CA Cancer J Clin 67: 7-30, 2017.

2. Torre LA, Siegel RL and Jemal A: Lung cancer statistics. Adv Exp Med Biol 893: 1-19, 2016.

3. Shi L, Tang J, Tong L and Liu Z: Risk of interstitial lung disease with gefitinib and erlotinib in advanced non-small cell lung cancer: A systematic review and meta-analysis of clinical trials. Lung Cancer 83: 231-239, 2014.

4. Li H, Schmid-Bindert G, Wang D, Zhao Y, Yang X, Su B and Zhou C: Blocking the PI3K/AKT and MEK/ERK signaling pathways can overcome gefitinib-resistance in non-small cell lung cancer cell lines. Adv Med Sci 56: 275-284, 2011.

5. Rolff J, Becker M, Merk J, Hoffmann J and Fichtner I: Preclinical study of a combination of erlotinib and bevacizumab in early stages of unselected non-small cell lung cancer patient-derived xenografts. Target Oncol 11: 507-514, 2016.

6. Lin L and Bivona TG: Mechanisms of resistance to epidermal growth factor receptor inhibitors and novel therapeutic strategies to overcome resistance in NSCLC patients. Chemother Res Pract 2012: 817297, 2012.

7. Sato M, Shames DS and Hasegawa Y: Emerging evidence of epithelial-to-mesenchymal transition in lung carcinogenesis. Respirology 17: 1048-1059, 2012.

8. Voena C, Varesio LM, Zhang L, Menotti M, Poggio T, Panizza E, Wang Q, Minero VG, Fagoonee S, Compagno M, et al: Oncogenic ALK regulates EMT in non-small cell lung carcinoma through repression of the epithelial splicing regulatory protein 1 . Oncotarget 7: 33316-33330, 2016.

9. Wu F, Li J, Jang C, Wang J and Xiong J: The role of Axl in drug resistance and epithelial-to-mesenchymal transition of non-small cell lung carcinoma. Int J Clin Exp Pathol 7: 6653-6661, 2014.

10. Jiao D, Wang J, Lu W, Tang X, Chen J, Mou H and Chen QY: Curcumin inhibited HGF-induced EMT and angiogenesis through regulating c-Met dependent PI3K/Akt/mTOR signaling pathways in lung cancer. Mol Ther Oncolytics 3: 16018, 2016.

11. Liu ZC, Chen XH, Song HX, Wang HS, Zhang G, Wang H, Chen DY, Fang R, Liu H, Cai SH and Du J: Snail regulated by PKC/GSK-3 $\beta$ pathway is crucial for EGF-induced epithelial-mesenchymal transition (EMT) of cancer cells. Cell Tissue Res 358: 491-502, 2014. 
12. Bi WR, Yang CQ and Shi Q: Transforming growth factor- $\beta 1$ induced epithelial-mesenchymal transition in hepatic fibrosis. Hepatogastroenterology 59: 1960-1963, 2012.

13. Kaminska B, Wesolowska A and Danilkiewicz M: TGF beta signalling and its role in tumour pathogenesis. Acta Biochim Pol 52: 329-337, 2005.

14. Shintani Y, Okimura A, Sato K, Nakagiri T, Kadota Y, Inoue M, Sawabata N, Minami M, Ikeda N, Kawahara K, et al: Epithelial to mesenchymal transition is a determinant of sensitivity to chemoradiotherapy in non-small cell lung cancer. Ann Thorac Surg 92: 1794-1804; discussion 1804, 2011.

15. Chen XF, Zhang HJ, Wang HB, Zhu J, Zhou WY, Zhang H, Zhao MC, Su JM, Gao W, Zhang L, et al: Transforming growth factor- $\beta 1$ induces epithelial-to-mesenchymal transition in human lung cancer cells via PI3K/Akt and MEK/Erk1/2 signaling pathways. Mol Biol Rep 39: 3549-3556, 2012.

16. Zhang HJ, Wang HY, Zhang HT, Su JM, Zhu J, Wang HB, Zhou WY, Zhang H, Zhao MC, Zhang L and Chen XF: Transforming growth factor- $\beta 1$ promotes lung adenocarcinoma invasion and metastasis by epithelial-to-mesenchymal transition. Mol Cell Biochem 355: 309-314, 2011.

17. Cervantes-Arias A, Pang LY and Argyle DJ: Epithelialmesenchymal transition as a fundamental mechanism underlying the cancer phenotype. Vet Comp Oncol 11: 169-184, 2013.

18. Keir ME, Butte MJ, Freeman GJ and Sharpe AH: PD-1 and its ligands in tolerance and immunity. Annu Rev Immunol 26 677-704, 2008

19. Dai S, Jia R, Zhang X, Fang Q and Huang L: The PD-1/PD-Ls pathway and autoimmune diseases. Cell Immunol 290: 72-79, 2014.

20. Bachy E and Coiffier B: Anti-PD1 antibody: A new approach to treatment of lymphomas. Lancet Oncol 15: 7-8, 2014.

21. Gunturi A and McDermott DF: Potential of new therapies like anti-PD1 in kidney cancer. Curr Treat Options Oncol 15: 137-146, 2014.

22. Kudo-Saito C, Shirako H, Takeuchi T and Kawakami Y: Cancer metastasis is accelerated through immunosuppression during Snail-induced EMT of cancer cells. Cancer Cell 15: 195-206, 2009.

23. Ye LY, Chen W, Bai XL, Xu XY, Zhang Q, Xia XF, Sun X, Li GG, Hu QD, Fu QH and Liang TB: Hypoxia-induced epithelial-to-mesenchymal transition in hepatocellular carcinoma induces an immunosuppressive tumor microenvironment to promote metastasis. Cancer Res 76: 818-830, 2016.

24. Li F, Zhu T, Cao B, Wang J and Liang L: Apatinib enchance antitumor activity of EGFR-TKIs in non-small cell lung cancer with EGFR-TKI resistance. Eur J Cancer 84: 184-192, 2017.

25. Hellmann M, Rizvi N, Wolchok JD and Chan TA: Genomic profile, smoking, and response to anti-PD-1 therapy in non-small cell lung carcinoma. Mol Cell Oncol 3: e1048929, 2015.
26. Riihimäki M, Hemminki A, Fallah M, Thomsen H, Sundquist K, Sundquist $\mathrm{J}$ and Hemminki $\mathrm{K}$ : Metastatic sites and survival in lung cancer. Lung Cancer 86: 78-84, 2014.

27. Singh D, Attri BK, Gill RK and Bariwal J: Review on EGFR Inhibitors: Critical updates. Mini Rev Med Chem 16: 1134-1166, 2016.

28. Nurwidya F, Takahashi F, Murakami A, Kobayashi I, Kato M, Shukuya T, Tajima K, Shimada N and Takahashi K: Acquired resistance of non-small cell lung cancer to epidermal growth factor receptor tyrosine kinase inhibitors. Respir Investig 52: 82-91, 2014.

29. Zhang Z, Lee JC, Lin L, Olivas V, Au V, LaFramboise T, Abdel-Rahman M, Wang X, Levine AD, Rho JK, et al: Activation of the AXL kinase causes resistance to EGFR-targeted therapy in lung cancer. Nat Genet 44: 852-860, 2012.

30. Nurwidya F, Takahashi F, Murakami A and Takahashi K: Epithelial mesenchymal transition in drug resistance and metastasis of lung cancer. Cancer Res Treat 44: 151-156, 2012.

31. Sequist LV, Waltman BA, Dias-Santagata D, Digumarthy S, Turke AB, Fidias P, Bergethon K, Shaw AT, Gettinger S, Cosper AK, et al: Genotypic and histological evolution of lung cancers acquiring resistance to EGFR inhibitors. Sci Transl Med 3: 75ra26, 2011.

32. Saijo N: Present status and problems on molecular targeted therapy of cancer. Cancer Res Treat 44: 1-10, 2012.

33. Zheng X, Carstens JL, Kim J, Scheible M, Kaye J, Sugimoto H, Wu CC, LeBleu VS and Kalluri R: Epithelial-to-mesemchymal transition is dispensable for metastasis but induces chemoresistance in pancreatic cancer. Nature 527: 525-530, 2015.

34. Diepenbruck M and Christofori G: Epithelial to mesemchymal transition (EMT) and metastais: Yes, no, maybe? Curr Opin Cell Biol 43: 7-13, 2016.

35. Strippoli R, Benedicto I, Perez Lozano ML, Pellinen T, Sandoval P, Lopez-Cabrera M and del Pozo MA: Inhibition of transforming growth factor-activated kinase 1 (TAK1) blocks and reverses epithelial to mesenchymal transition of mesothelial cells. PLoS One 7: e31492, 2012.

36. Liu W, Kovacevic Z, Peng Z, Jin R, Wang P, Yue F, Zheng M, Huang ML, Jansson PJ, Richardson V, et al: The molecular effect of metastasis suppressors on Src signaling and tumorigenesis: New therapeutic targets. Oncotarget 6: 35522-35541, 2015.

37. Sequist LV, Bell DW, Lynch TJ and Haber DA: Molecular predictors of response to epidermal growth factor receptor antagonists in non-small-cell lung cancer. J Clin Oncol 25: 587-595, 2007.

38. Said NA and Williams ED: Growth factors in induction of epithelial-mesenchymal transition and metastasis. Cells Tissues Organs 193: 85-97, 2011. 